ИЗВЕСТИЯ АКАДЕМИИ НАУК ЭСТОНСКОП ССР. ФИЗИКА. МАТЕМАТИКА

PROCEEDINGS OF THE ACADEMY OF SCIENCES OF THE ESTONIAN SSR.

PHYSICS. MATHEMATICS

$1984,33,2$

\title{
Ингрид МАУЭР
}

\section{ОБ ОДНОМ СПОСОБЕ ПОСТРРОЕНИЯ ТЕСТОВЫХ ЗАДАЧ ДЛЯ АЛГОРИТМОВ МИНИМИЗАЦИИ}

\author{
(Представил Н. Алумяэ)
}

Результаты практических и теоретических исследований показывают, что скорость локальной сходимости алгоритмов при решении задач безусловной оптимизации значительно зависит от числа обусловленности гессиана оптимизируемой функции в точке экстремума, причем трудности решения, как правило, увеличиваются с ростом этого числа. Поэтому понятие числа обусловленности широко используется при построении тестовых задач для многих алгоритмов безусловной оптимизации. Но для составления условных тестовых задач наряду с целевой функцией необходимо учитывать и ограничения задачи. Так, в $\left[{ }^{1}\right]$ при составлении условных задач, аналогично числу обусловленности в безусловной оптимизации, предлагают использовать число обусловленности некоторого уменьшенного гессиана функции Лагранжа составляемой задачи в точке ее решения и рассматривать это число как меру трудности этой задачи.

В данной работе описан базирующийся на этой мере способ построения условных и безусловных тестовых задач минимизации. Составляемые этим способом задачи содержат параметр, с ростом которого к бесконечности стремится к бесконечности и отмеченное число обусловленности. Таким образом, данный способ характеризуется целенаправленностью, без которой, как аргументируется в $\left[{ }^{2}\right]$, составляемые условные задачи имеют тенденцию быть по предложенной в $\left[{ }^{1}\right]$ мере сравнительно хорошо обусловленными.

Построим задачу

$$
\min \left\{f(x) \mid g_{1}(x) \leqslant 0, g_{2}(x)=0\right\},
$$

где $x \in E^{n}$ и функция $f(x)$ и вектор-функции $g_{1}(x) \quad\left(m_{1}\right.$-мерная) и $g_{2}(x)$ $\left(m_{2}\right.$-мерная) дважды непрерывно дифференцируемые. Обозначим через $f_{x}(\bar{x})$ и $f_{x x}(\bar{x})$ соответственно градиент и гессиан функции $f(x)$ в точке $\bar{x}$.

Пусть для этой задачи известны решение $x^{*}$ и точка $u^{*}=$ $=\left(u_{1}^{*}, u_{2}^{*}\right), \quad u_{1} \in E^{m_{1}}, \quad u_{2} \in E^{m_{2}}, u \in E^{m_{1}+m_{2}}, \quad$ такая, что пара $\left(x^{*}, u^{*}\right)$ удовлетворяет необходимым условиям Куна-Таккера, т. е. $g_{1}\left(x^{*}\right) \leqslant 0$, $g_{2}\left(x^{*}\right)=0, \quad u_{1}{ }^{*} \geqslant 0, \quad u_{1}{ }^{* \top} g_{1}\left(x^{*}\right)=0, L_{x}\left(x^{*}, u^{*}\right)=0, \quad$ где $L(x, u)-$ функция Лагранжа задачи (1).

Пусть в точке $x^{*}$ опорные гиперплоскости всех активных ограничений задачи $(1)$, за исключением тех из $g_{1}(x) \leqslant 0$, которым в $u_{1}{ }^{*}$ соответствуют нулевые компоненты, выражаются системой уравнений

$$
A\left(x-x^{*}\right)=0 .
$$

Строками матрицы $A$ являются градиенты соответствующих компонент $g_{1}(x)$ и $g_{2}(x)$ в точке $x^{*}$. 
Предположим, что строки матрицы $A$ линейно независимые, причем их число $m<n$, а пара $\left(x^{*}, u^{*}\right)$ удовлетворяет условию второго порядка: для любого вектора $z \neq 0\left(z \in E^{n}\right), A z=0$ имеет место

$$
z^{\mathrm{T}} L_{x x}\left(x^{*}, u^{*}\right) z>0 .
$$

При выполнении этих условий $x^{*}$ - изолированное локальное решеные задачи (1) и $u^{*}$ определена единственным образом (см. $\left.\left[{ }^{1,3}\right]\right)$. Отметим, что такую задачу можно построить, например, изложенными в $[4,5]$ способами.

Составим теперь новую задачу с параметром; слагая к целевой функции построенной задачи (1) некоторую функцию,

$$
\min _{x}\left\{f(x)+\frac{1}{2} t\left\|v(x)-v\left(x^{*}\right)\right\|^{2} \mid g_{1}(x) \leqslant 0, g_{2}(x)=0\right\} .
$$

Здесь $v(x)$ - дважды непрерывно дифференцируемая $s$-мерная векторфункция, $t$ - неотрицательный скалярный параметр и норма - евклидова пространства.

При любом значении $t \leqslant 0$, как нетрудно убедиться, точка $x^{*}$ является изолированным локальным решением задачи (2) и пара $\left(x^{*}, u^{*}\right)$ удовлетворяет необходимым условиям Куна-Таккера этой задачи, причем $u^{*}$ единственно определена.

Обозначим функцию Лагранжа задачи (2) через $\mathfrak{S}(x, u ; t)$.

Не ограничивая общности, можно считать, что первые $m$ столбцов матрицы $A$ линейно независимые. Пусть матрица $A=(B, C)$, где $B-$ $(m \times m)$-мерная и $C-(m \times(n-m))$-мерная матрицы. Так как строки матрицы $Z^{\mathrm{T}}=\left(\left(-B^{-1} C\right)^{\mathrm{T}}, I\right)$, где $I-((n-m) \times(n-m))$-мерная единичная матрица, линейно независимые и $A Z=0$, то с помощью этих строк можно получить $n-m$ ортонормированных векторов, определяющих пространство $D=\{z \mid A z=0\}$. Пусть эти векторы являются столбцами $(n \times(n-m))$-мерной матришы $Q$.

Запишем теперь матрицу $\mathfrak{I}_{x x}\left(x^{*}, u^{*} ; t\right)$, уменьшенную на пространство $D$,

$$
Q^{\mathrm{T}} \Re_{x x}\left(x^{*}, u^{*} ; t\right) Q
$$

и обозначим через $p(t)$ функцию, значение которой при произвольной величине $\bar{t} \geqslant 0$ аргумента $t \div$ число обусловленности этой матрицы при $t=\bar{t}$.

По предложенному в $\left[{ }^{1}\right]$, мерой трудности задачи (2) со значением $\bar{t}$ параметра $t$ можно рассматривать число $p(\bar{t})$ и, следовательно, тестовыми задачами подходят такие задачи (2) с фиксированным параметром, для которых это число сравнительно большое. Таким образом, для получения хороших тестовых задач предложим в задаче (2) определить вектор-функцию $v(x)$ такой, чтобы для этой задачи $p(t) \rightarrow \infty$ при $t \rightarrow \infty$. В последующем приведем необходимые и достаточные условия, при выполнении которых этот предельный переход имеет место.

Далее используем замену переменных $x=Q y+x^{*}, y \in E^{n-m}$, которая определяет однозначное соответствие между точками множества $\bar{D}=\left\{x \mid A\left(x-x^{*}\right)=0\right\}$ и пространства $E^{n-m}$ и, в частности, точке $x^{*}$ соответствует точка $y^{*}=0$. Но, если $D=\bar{D}$, т. е. $A x^{*}=0$, то можно использовать и замену $x=Q y$, в случае которой $x^{*}=Q y^{*}$.

Введем вспомогательную функцию

для которой

$$
\Phi(y ; t)=\mathfrak{Q}\left(Q y+x^{*}, u^{*} ; t\right),
$$

$$
\Phi_{y y}(y ; t)=Q^{\mathrm{T}}{ }_{x x}\left(Q y+x^{*}, u^{*} ; t\right) Q,
$$


и, в частности,

$$
\Phi_{y y}\left(y^{*} ; t\right)=Q^{\mathrm{T}} \mathfrak{\Omega}_{x x}\left(x^{*}, u^{*} ; t\right) Q .
$$

Обозначим через $V$ якобиан вектор-функции $v(x)$ в точке $x^{*}$ и покажем, что при любом значении $t \geqslant 0$ матрица $\Phi_{y y}\left(y^{*} ; t\right)$ положительно определена. Действительно, для любой точки $y \neq 0$ величина

$$
\begin{gathered}
y^{\mathrm{T}} \Phi_{y y}\left(y^{*} ; t\right) y=y^{\mathrm{T}} Q^{\mathrm{T}} L_{x x}\left(x^{*}, u^{*}\right) Q y+t y^{\mathrm{T}} Q^{\mathrm{T}} V^{\mathrm{T}} V Q y= \\
=z^{\mathrm{T}} L_{x x}\left(x^{*}, u^{*}\right) z+t z^{\mathrm{T}} V^{\mathrm{T}} V z
\end{gathered}
$$

положительная; это по условию второго порядка задачи (1), поскольку $z \neq 0, A z=0$, и симметричности матрицы $V^{\mathrm{T}} V$.

Теперь можно рассмотреть функцию

$$
p(t)=\left\|\Phi_{y y}\left(y^{*} ; t\right)\right\|\left\|\left(\Phi_{y y}\left(y^{*} ; t\right)\right)^{-1}\right\| .
$$

В этой функции при $t \geqslant 0$ обратная матрица от $\Phi_{y y}\left(y^{*} ; t\right)$ всегда существует, так как эта матрица при любом значении $t \geqslant 0$ положительно определена.

Введем обозначение

$$
W=Q^{\mathrm{T}} V^{\mathrm{T}} V Q
$$

Так как абсолютная величина определителя не изменится при перестановке его строк (столбцов) и при сложении к одной строке (столбцу) умноженной на пронзвольное число другой строки (столбец), то этими преобразованиями наряду с матрицей $\Phi_{y y}\left(y^{*} ; t\right)=\Phi_{y y}\left(y^{*} ; 0\right)+t W$ можно найти и другую матрицу того же типа, в которой вместо $\Phi_{y y}\left(y^{*} ; 0\right)$ имеется другая независящая от $t$ матрица, а вместо $W$ диагональная матрица (если, разумеется, матрица W сама уже не является диагональной), и такую, что их определители совпадают до знака (см. $\left.\left[{ }^{6}\right]\right)$. Число отличных от нуля элементов в полученной вместо $W$ диагональной матрице является рангом матрицы $W$. Следовательно, определитель матрицы $\Phi_{y y}\left(y^{*} ; t\right)-$ многочлен от $t$, степень которого равна рангу матрицы $W$.

Т ео рем а. Для того, чтобы

$$
p(t) \rightarrow \infty
$$

при $t \rightarrow \infty$, необходимо и достаточно, чтобы матрица $V$ удовлетворяла условиям

a) $V \neq 0$,

б) $W$ вырожденная.

Доказ ательство. Необходимость. Для того, чтобы $p(t) \rightarrow \infty$ при $t \rightarrow \infty$, необходимо, чтобы $W \neq 0$, но это невозможно, если $V=0$. Также необходимо, чтобы W была вырожденной. В самом деле, если матрица $W$ невырожденная, то при $t \rightarrow \infty$ функция $p(t)$

$$
\left\|\frac{1}{t} \Phi_{y y}\left(y^{*} ; t\right)\right\|\left\|\left(\frac{1}{t} \Phi_{y y}\left(y^{*} ; t\right)\right)^{-1}\right\| \rightarrow\|W\|\|W-1\|,
$$

которое конечная величина.

Достаточность. Пусть выполняются условия а) и б) теоремы. Из-за $V \neq 0$ можно найти точку $\bar{z}$ такую, что $V \bar{z} \neq 0$. Определяя еще точку $\bar{y}$, для которой $\bar{z}=Q \bar{y}$, получим, что $\bar{z}^{\mathrm{T}} V^{\mathrm{T}} V \bar{z}=\bar{y}^{\mathrm{T}} W \bar{y}>0$, откуда вытекает, что $W \neq 0$.

Знаменатель, т. е. определитель матрицы $\Phi_{y y}\left(y^{*} ; t\right)$, и числитель, т. е. соответствующее рассматриваемому элементу алгебраическое дополнение той же матрицы всех элементов матрицы $\left(\Phi_{y y}\left(y^{*} ; t\right)\right)^{-1}$, 
являются многочленами от $t$. Определитель матрицы $\Phi_{y y}\left(y^{*} ; t\right)$, т. е. знаменатель всех элементов $\left(\Phi_{y y}\left(y^{*} ; t\right)\right)^{-1}$, как мы уже показали, многочлен, степень которого равна рангу матрицы $W$. Но по величине ту же степень имеет и числитель хоть одного элемента матрицы $\left(\Phi_{y y}\left(y^{*} ; t\right)\right)^{-1}$; в этом можно аналогично убедиться, учитывая, что матрица $\mathbb{W}$ вырожденная. По такому анализу элементов матрицы $\left(\Phi_{y y}\left(y^{*} ; t\right)\right)^{-1}$ приходим к выводу, что предельная матрица здесь при $t \rightarrow \infty$ не является нулевой и, следовательно, $\left\|\left(\Phi_{y y}\left(y^{*} ; t\right)\right)^{-1}\right\|$ стремится к отличному от нуля пределу при $t \rightarrow \infty$. В то же время $\left\|1 / t \Phi_{y y}\left(y^{*} ; t\right)\right\| \rightarrow$ $\rightarrow\|W\|$, которая также отличается от нуля, так как $W \neq 0$. Следовательно, функция $p(t)$

$$
t\left\|\frac{1}{t} \Phi_{y y}\left(y^{*} ; t\right)\right\|\left\|\left(\Phi_{y y}\left(y^{*} ; t\right)\right)^{-1}\right\| \rightarrow \infty
$$

при $t \rightarrow \infty$. Теорема доказана.

Так как ранг произведения матриц не превосходит рангов множителей этого произведения, то для выполнения условия б) теюремы достаточно выбрать числа $m, n$ и $s$ составляемой задачи такими, чтобы имело место неравенство $n-m>s$.

Однако не все задачи (2), вектор-функция $v(x)$ которых удовлетворяет условиям теоремы, с одинаковым успехом применимы в практике. Поэтому обратим внимание на функцию $\Phi(y ; t)$. Так как $\Phi_{y}\left(y^{*} ; t\right)=$ $=Q^{\mathrm{T}} L_{x}\left(x^{*}, u^{*}\right)=0$, поскольку $L_{x}\left(x^{*}, u^{*}\right)=0$, и матрица $\Phi_{y y}\left(y^{*} ; t\right)$ при любом значении $t \geqslant 0$ положительно определена, то для всех $t \geqslant 0$ точка $y^{*}$ - изолированный локальный минимум этой функции. Опираясь на это обстоятельство, можно предложить в функции Ф $(y ; t)$ при фиксированном значении $t>0$ выбрать вектор-функцию $v\left(Q y+x^{*}\right)$ такой, чтобы условия теоремы были выполнены и одновременно функция $Ф(y ; t)$ имела в окрестности точки $y^{*}$ такую овражность, которая с ростом $t$ стала сразу быстро увеличиваться, а затем эту вектор-функцию перенести в виде $v(x)$ в задачу (2).

Проиллюстрируем этот способ примерами безусловных задач (2), для которых $x=\left(x_{1}, x_{2}\right)^{\mathrm{T}} \in E^{2}, x^{*}=0, Q=Z=I(m=0), D=\bar{D}=E^{2}$, $x=y$. Рассмотрим задачи

a) $\min _{x} \frac{1}{2}\left[x_{1}^{2}+x_{2}^{2}+t\left(x_{1}+x_{2}\right)^{2}\right], \quad t \geqslant 0$,

где $V=(1,1), V^{\mathrm{T}} V$ вырожденная, $p(t)=2 t+1$;

б) $\min _{x} \frac{1}{2}\left[x_{1}^{2}+x_{2}^{2}+100\left(x_{1}+x_{2}\right)^{2}+t\left(x_{1}-x_{2}\right)^{2}\right], t \geqslant 0$,

где $V=(1,1), V^{\mathrm{T}} V$ вырожденная, $p(t)=2 t+1$;

$$
p(t)= \begin{cases}\frac{201}{2 t+1}, & t \in[0,100] \\ \frac{2 t+1}{201}, & t \in(100, \infty) .\end{cases}
$$

В примере а) с ростом $t$ овражность минимизируемой функции увеличивается, но в примере б) овражность сначала уменьшается, а начиная со значения $t=100$ увеличивается.

Автор благодарит О. Ваарманна за ценные замечания. 
1. Hock, W., Schittkowski, K. Lect. Notes Econ. and Math. Syst., № 187 (1981).

2. Stewart, G. H. Math. Programming, 21, № 2, 235-238 (1981).

3. Фиакко А., Мак-Кормик $Г$. Нелинейное программирование. Методы последовательной безусловной минимизации. М., «Мир» 1972.

4. Schittkowski, K. Lect. Notes Econ. and Math. Syst., № 183, (1980).

5. Rosen, J. B., Suzuki, S. Communs ACM, 8, № 2, 113, (1965).

6. Kangro, G. Kõrgem algebra. Tallinn, ERK. 1962.

Институт кибернетики

Академии наук Эстонской ССР

\section{Поступила в редакцию} 17/XI 1982

\section{Ingrid MAUER | \\ UHEST MINIMEERIMISALGORITMIDELE TESTULESANNETE KOOSTAMISE VIISIST}

Artiklis on koostatud parameetrit sisaldav ülesanne (2) ning näidatud, et teatud tingimustel parameetri kasvades lōpmatusse suureneb lōpmatusse ka selle ülesande raskusmõõduna vaadeldav arv.

\section{Ingrid MAUER.}

\section{ABOUT A WAY OF CONSTRUCTING TEST PROBLEMS FOR ALGORITHMS OF MINIMIZATION}

In this paper a problem, including the parameter, has been constructed (problem (2)). It is shown that under certain conditions, if the parameter is increasing to infinity, the condition figure considered as a measure of difficulty for the given problem, increases to infinity, too. 